SLAC-PUB-8742

BABAR-PROC-00/31

hep-ex/0101035

January, 2001

\title{
Physics at BABAR
}

\author{
Christos Touramanis \\ University of Liverpool \\ Department of Physics, Oliver Lodge Laboratory \\ L69 7ZE, Liverpool, U.K. \\ (for the BABAR Collaboration)
}

\begin{abstract}
The BABAR detector at the SLAC PEP-II asymmetric $e^{+} e^{-}$collider has first started collecting data in May 1999. A study of time-dependent $C P$-violating asymmetries in $B^{0} \rightarrow J / \psi K_{S}^{0}$ and $B^{0} \rightarrow \psi(2 S) K_{S}^{0}$ decays has been performed on a data sample of $9.0 \mathrm{fb}^{-1}$ taken at the $\Upsilon(4 S)$ resonance and $0.8 \mathrm{fb}^{-1}$ off-resonance, collected through July 2000 . The preliminary result $\sin 2 \beta=$ $0.12 \pm 0.37$ (stat) \pm 0.09 (syst) is presented here, together with preliminary results on neutral and charged $B$ meson lifetimes and $B^{0} \bar{B}^{0}$ mixing.
\end{abstract}

5th International Symposium on Radiative Corrections

(RADCOR-2000)

Carmel CA, USA, 11-15 September, 2000

Stanford Linear Accelerator Center, Stanford University, Stanford, CA 94309

Work supported in part by the U.K. Particle Physics and Astronomy Research Council, Advanced

Research Fellowship GR/L04177 and Department of Energy contract DE-AC03-76SF00515. 


\section{Introduction}

The three-generation Standard Model can accommodate $C P$ violation through the presence of a non-zero imaginary phase in the Cabibbo-Kobayashi-Maskawa (CKM) quark mixing matrix. However, existing measurements of $C P$ violation in the neutral kaon system cannot prove that the CKM phase is indeed the origin of $C P$ violation in nature.

The primary goal of the BABAR experiment at PEP-II is to elucidate this question by a series of observations and measurements of $C P$-violating effects in the $B$ meson system. These measurements allow the extraction of the angles $\alpha, \beta$ and $\gamma$ of the Unitarity Triangle, whose non-zero area [1] is a direct measure of $C P$ violation.

$B A B A R$ can also access the sides of the Unitarity Triangle through measurements of $\left|V_{u b}\right|,\left|V_{c b}\right|$ in semileptonic $B$ decays and $\left|V_{t d}\right|$ in $B^{0} \bar{B}^{0}$ mixing. This allows to overconstrain the Unitarity Triangle and perform stringent tests of the Standard Model.

Thus, high statistics, a clean environment and broad access to the rich phenomenology of the $B$ sector will allow $B A B A R$ to improve our knowledge of the overall $B$ decay picture and probe New Physics at higher energy scales. A broad heavy flavor physics programme is also ongoing in BABAR.

\section{PEP-II}

The PEP-II $B$ Factory [2] is an $e^{+} e^{-}$colliding beam storage ring complex at SLAC designed to produce a luminosity of $3 \times 10^{33} \mathrm{~cm}^{-2} \mathrm{~s}^{-1}$ at a center-of-mass energy of $10.58 \mathrm{GeV}(\Upsilon(4 S)$ resonance). During the 2000 run PEP-II has exceeded this luminosity, while BABAR, with a logging efficiency of $>95 \%$, has routinely accumulated data above its design daily rate of $135 \mathrm{pb}^{-1}$.

The machine has asymmetric energy beams, with a High Energy Ring (HER, 9.0 GeV electrons) and a Low Energy Ring (LER, 3.1 GeV positrons). These correspond to a center-of-mass boost of $\beta \gamma=0.56$ and lead to an average separation of $\beta \gamma \mathrm{c} \tau=250 \mu \mathrm{m}$ between the two $B$ mesons vertices, allowing the measurement of time-dependent $C P$-violating decay rate asymmetries.

At the $\Upsilon(4 S)$ resonance $B$ mesons can only be produced as $B^{+} B^{-}$or coherent $B^{0} \bar{B}^{0}$ pairs. The time evolution of a coherent $B^{0} \bar{B}^{0}$ pair is coupled in such a way that the $C P$ or flavor of one $B$ at decay time $t_{1}$ can be described as a function of the other $B\left(B_{\text {tag }}\right)$ flavor at its decay time $t_{2}$ and the signed time difference $\Delta t=t_{1}-t_{2}$.

\section{$3 \quad$ BABAR}

\subsection{Detector description [2]}

The volume within the $1.5 \mathrm{~T}$ BABAR superconducting solenoid contains a five layer silicon strip vertex detector (SVT), a central drift chamber (DCH), a quartz-bar Cherenkov radiation detector (DIRC) and a CsI(Tl) crystal electromagnetic calorimeter (EMC). Two layers of cylindrical resistive plate counters (RPCs) are located between the barrel calorimeter and the magnet cryostat. The instrumented flux return (IFR) outside the cryostat is composed of 18 layers of radially increasing thickness steel, instrumented with 19 layers of planar RPCs in the barrel and 18 in the endcaps which provide muon and neutral hadron identification. 


\subsection{Particle reconstruction and identification [2]}

Charged particle tracking using the SVT and DCH achieves a resolution of $\left(\delta p_{T} / p_{T}\right)^{2}=\left(0.0015 p_{T}\right)^{2}+$ $(0.005)^{2}$, where $p_{T}$ is the transverse momentum in $\mathrm{GeV} / c$. The SVT with a typical resolution of $10 \mu \mathrm{m}$ per hit provides excellent vertex resolution both in the transverse plane and in $z$. The typical fully reconstructed single $B$ decay vertex resolution in $z$ is $50 \mu \mathrm{m}$. Photons are reconstructed in the EMC, yielding mass resolutions of $6.9 \mathrm{MeV} / c^{2}$ for $\pi^{0} \rightarrow \gamma \gamma$ and $10 \mathrm{MeV} / c^{2}$ for $K_{S}^{0} \rightarrow \pi^{0} \pi^{0}$.

Leptons and hadrons are identified using a combination of measurements from all the BABAR components, including the energy loss $\mathrm{d} E / \mathrm{d} x$ in the helium-based gas of the DCH (40 samples maximum) and in the silicon of the SVT (5 samples maximum). Electron identification is mainly based on the characteristics of their shower in the EMC, while muons are identified in the IFR and confirmed by their minimum ionising signal in the EMC. Excellent kaon identification in the barrel region is provided by the DIRC, which achieves a separation of $>3.4 \sigma$ in the range $0.25-3.5 \mathrm{GeV} / c$.

\section{$4 \quad B$ reconstruction}

A variety of inclusive, semiexclusive and exclusive reconstruction methods are applied on the BABAR data, covering semileptonic and pure hadronic decay modes. The corresponding $B$ samples have different sizes and purity levels and are used for different types of studies (Branching Fraction measurements, studies of the dynamics of certain decay chains). We will focus here on the cases where some information (final state(s), charge, $C P$ or flavor content, decay vertex) can be reconstructed for both $B$ mesons in the event.

\subsection{Exclusive $B$ sample}

$B^{0}$ and $B^{ \pm}$mesons are reconstructed in the following hadronic modes of definite flavor: $B^{0} \rightarrow$ $D^{(*)-} \pi^{+}, D^{(*)-} \rho^{+}, D^{(*)-} a_{1}^{+}, J / \psi K^{* 0}, B^{-} \rightarrow D^{0} \pi^{-}$and $B^{-} \rightarrow D^{* 0} \pi^{-1}$. All final state particles are reconstructed. The selections have been optimised for signal significance, using on-peak, offpeak and simulated data. Charged particle identification, mass(or mass difference) and vertex constraints are used wherever applicable. The signal for each decay mode is identified in the twodimensional distribution of the kinematical variables $\Delta E$ and $m_{\mathrm{ES}}: \Delta E=E_{\mathrm{rec}}^{*}-E_{b}^{*}$ is the difference between the $B$ candidate energy and the beam energy and $m_{\mathrm{ES}}=\sqrt{E_{b}^{* 2}-\boldsymbol{p}_{\text {rec }}^{* 2}}$ is the mass of a particle with a reconstructed momentum $\boldsymbol{p}_{\text {rec }}^{*}=\sum_{i} \boldsymbol{p}_{i}^{*}$ assumed to have the beam energy, as is the case for a true $B$ meson. In events with several $B$ candidates only the one with the smallest $\Delta E$ is considered. The $\Delta E$ and $m_{\mathrm{ES}}$ variables have minimal correlation. The resolution in $m_{\mathrm{ES}}$ is $\approx 3 \mathrm{MeV} / c^{2}$ and is dominated by the beam energy spread. The resolution in $\Delta E$ is mode dependent and varies in the range of $12-40 \mathrm{MeV}$. For each mode a rectangular signal region is defined by the three standard deviation bands in $m_{\mathrm{ES}}\left(5.27<m_{\mathrm{ES}}<5.29 \mathrm{GeV} / c^{2}\right)$ and $\Delta E$ (mode dependent interval). For each mode the sample composition is determined by fitting the $m_{\mathrm{ES}}$ distribution for candidates within the signal region in $\Delta E$ to the sum of a single Gaussian representing the signal and a background function introduced by the ARGUS collaboration [3]. The purity of each subsample is computed as the ratio of the area of the Gaussian in the $\pm 3 \sigma$ range over the total area in this range. Figure 1 shows the $m_{\mathrm{ES}}$ distributions for the summed hadronic $B^{0}$ and $B^{ \pm}$modes with the fits superimposed.

\footnotetext{
${ }^{1}$ Throught this paper, conjugate modes are implied.
} 

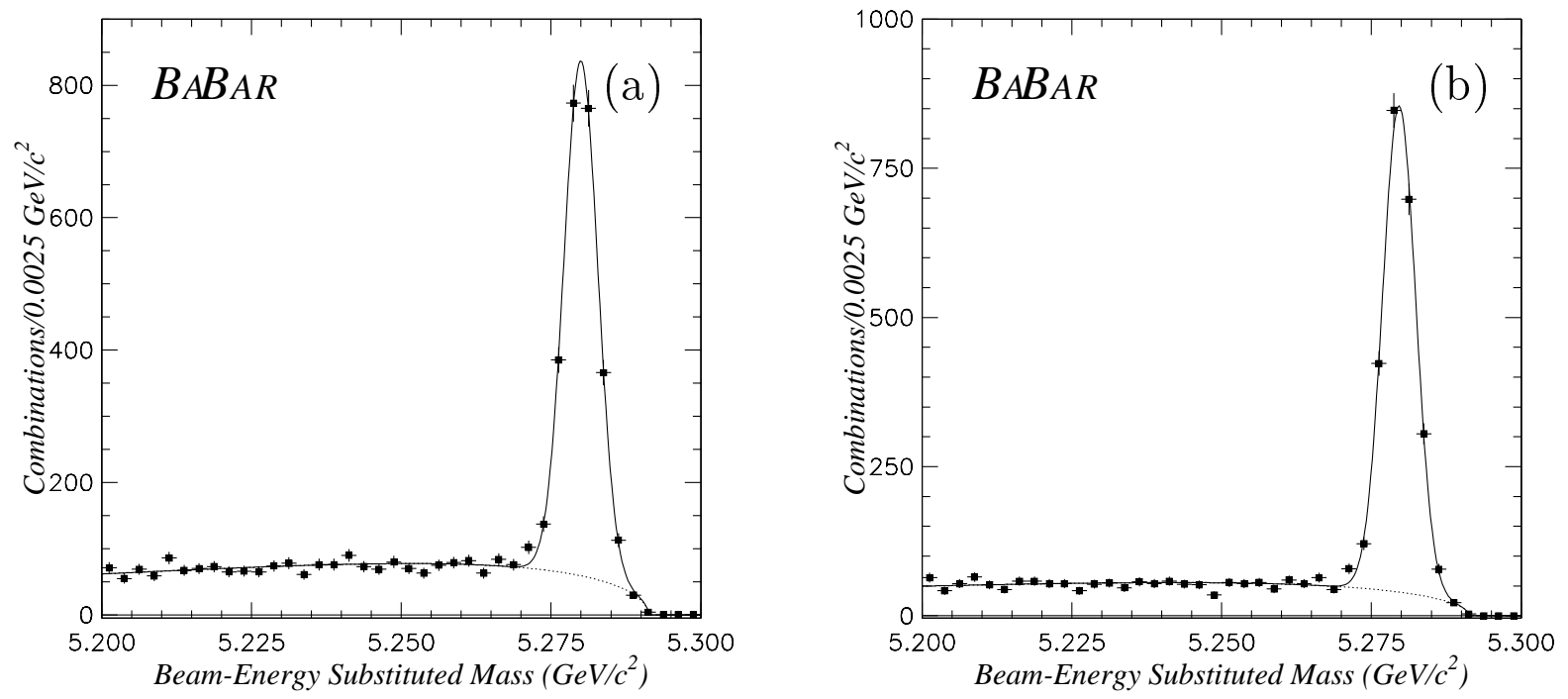

Figure 1: $m_{\mathrm{ES}}$ distribution for all the hadronic modes for (a) $B^{0}$ and (b) $B^{ \pm}$. The complete fit and its ARGUS [3] background content are also shown. The number of signal events in all $B^{0}$ and $B^{ \pm}$ modes are $2577 \pm 59$ and $2636 \pm 56$, with purity of $\approx 86 \%$ and $\approx 89 \%$ respectively.

\subsection{Flavor tagging}

After removal of the daughter tracks of the reconstructed $B_{\text {rec }}$ in an event, the remaining tracks are used to determine the flavor of the other $B$ meson $\left(B_{t a g}\right)$, and this ensemble is assigned a tag flavor, either $B^{0}$ or $\bar{B}^{0}$.

For each of the tagging methods used we define an effective tagging efficiency $Q_{i}=\varepsilon_{i} \times$ $\left(1-2 w_{i}\right)^{2}$, where $\varepsilon_{i}$ is the fraction of events tagged by this method $i$ and $w_{i}$ is the mistag fraction, i.e. the probability of incorrectly assigning the opposite tag to an event using this method. A dilution factor is defined as $\mathcal{D}=1-2 w$ and is extracted from the data for each method.

The Lepton category uses the presence and charge of a primary lepton from the decaying $b$ quark. Both electrons and muons are used, with a minimum center-of-mass momentum requirement of $1.1 \mathrm{GeV} / c$. If both an electron and a muon candidate satisfy this requirement, only the electron is taken into account. Mistag arises from (a) pions seen as leptons and (b) softer opposite-sign leptons coming from charm semileptonic decays.

The Kaon category is based on the total charge of all identified Kaons. Events with conflicting Lepton and Kaon tags are excluded from both categories.

For events not tagged with the previous methods, a variety of available particle identification and kinematic variables are fed in a Neural Network whose design and training aims at exploiting the information present in this set of correlated quantities. It is sensitive to the presence of primary and cascade leptons, charged kaons and soft pions from $D^{*}$ decays. In addition, the charge of highmomentum particles is exploited in a "jet-charge" type approach. This functionality has been assigned to different sub-nets, to facilitate understanding of the network performance. The output from the full neural network tagger $x_{N T}$ is mapped onto the interval $[-1,1]$. The assigned flavor 
$\operatorname{tag}$ is $B^{0}$ if $x_{N T}$ is negative, and $\bar{B}^{0}$ otherwise. Events with $\left|x_{N T}\right|>0.5$ are assigned to the NT1 tagging category and events with $0.2<\left|x_{N T}\right|<0.5$ to the NT2 tagging category. Events with $\left|x_{N T}\right|<0.2$ have very little tagging power and are rejected.

\section{3 $\Delta t$ calculation and resolution}

Since no stable charged particle emerges from the $\Upsilon(4 S)$ decay point, the production point of the $B$ mesons and thus their individual decay times cannot be determined. However the decay time difference $\Delta t$ between the two is sufficient for the description of a coherent $B$ meson pair (decay length difference technique).

The event topology is sketched in Fig. 2. In the boost approximation used in BABAR the decay time difference is calculated as : $\Delta t=\Delta z / c<\beta \gamma>$, where the small flight path of the $B$ mesons perpendicular to the $\mathrm{z}$ exis is ignored.

Actually, the small effects arising from the tilt of the PEP-II beams with respect to the BABAR z axis $(20 \mathrm{mr})$, fluctuations in the beam energies, the $B$ meson transverse momentum in the $\Upsilon(4 S)$ rest frame, have been studied and are taken into account either in the calculations or in the systematic errors as appropriate.

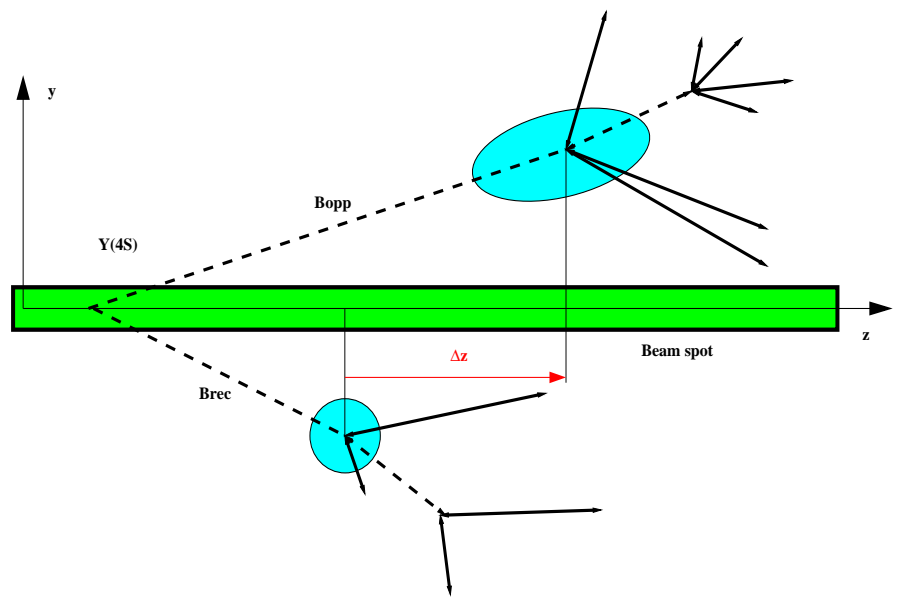

Figure 2: Event topology showing the two $B$ production and decay points. The figure is not drawn to scale; it has been expanded in the y direction.

The resolution $\sigma_{z}$ for the fully reconstructed $B$ is found in the simulation to be $45-65 \mu \mathrm{m}$, depending on the mode. The resolution $\sigma_{z}$ for the tag side is $\approx 125 \mu \mathrm{m}$, with a small bias of $25 \mu \mathrm{m}$ due to forward-going charm decays that cannot be resolved. The resulting resolution in $\Delta t$ has been parametrised as the sum of three gaussians. The core has a $\sigma$ of $0.6 \mathrm{ps}$ and contains $75 \%$ of the events. The tail has a $\sigma$ of $1.8 \mathrm{ps}$. Outliers are described by a gaussian with fixed $\sigma$ of $8 \mathrm{ps}$, that contains $\approx 1 \%$ of the total events. This resolution model is used for the lifetime, mixing and $\sin 2 \beta$ fits. Two scale factors (multiplicative to the width of the core and tail gaussians) are included in the fits to the real data for the first two cases, to account for eventual imperfections in the modeling of $D$ decays and multiple scattering in the simulation. Extensive studies on the different event samples and with variations of the fits (free and fixed parameters) have been performed in order to optimise and validate the method and to obtain reliable estimates of the systematic errors. 


\section{$5 \quad B$ lifetime measurements}

The observed $\Delta t$ distribution for a set of $B$ pair events in the presence of the resolution function $\mathcal{R}$ is :

$$
\mathcal{F}(\Delta t)=\Gamma \exp (-\Gamma|\Delta t|) \otimes \mathcal{R}(\Delta t ; \hat{a})
$$

where $\hat{a}$ is the set of parameters describing the resolution function.

The $B$ meson lifetimes are extracted with unbinned maximum likelihood fits that take individual event $\Delta t$ errors into account. Our preliminary results are :

$$
\begin{aligned}
\tau_{B^{0}} & =1.506 \pm 0.052(\text { stat }) \pm 0.029 \text { (syst) ps } \\
\tau_{B^{+}} & =1.602 \pm 0.049(\text { stat }) \pm 0.035 \text { (syst) ps } \\
\tau_{B^{+}} / \tau_{B^{0}} & =1.065 \pm 0.044 \text { (stat) } \pm 0.021 \text { (syst) }
\end{aligned}
$$

The only background source is combinatorial and it is estimated from the side-bands of the beam energy substituted mass variable. The main systematic error comes from the resolution modeling and parameters. The two proper time fits are shown in Figure 3.
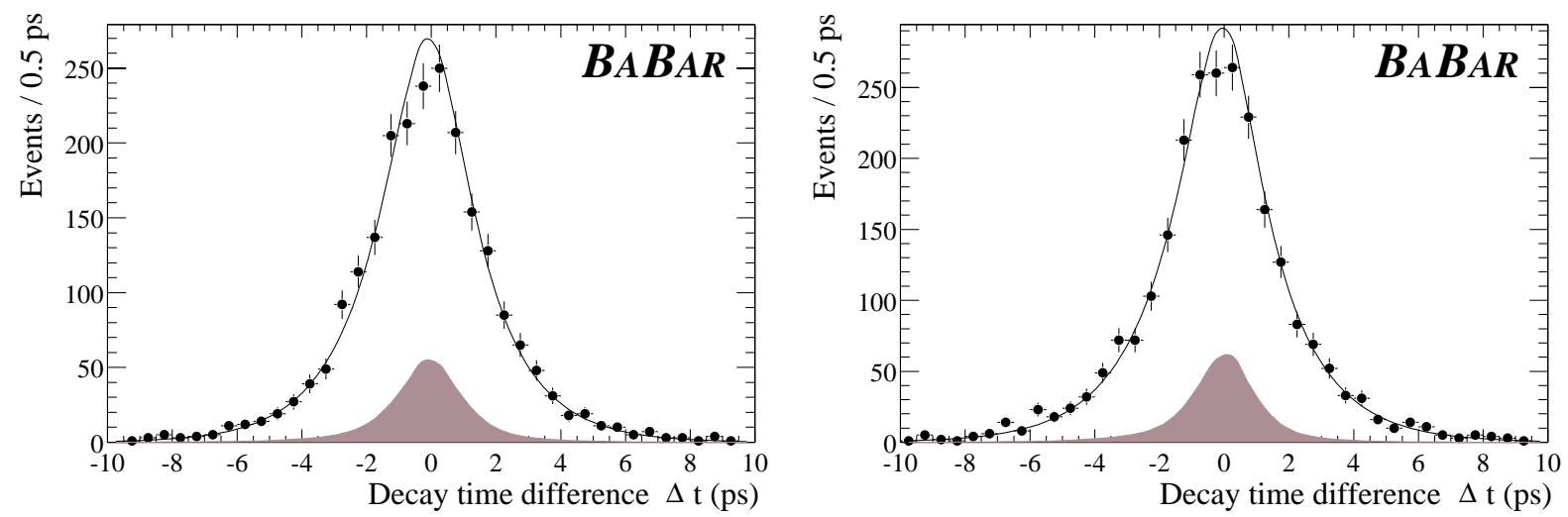

Figure 3: $\Delta t$ distributions for the $B^{0}$ (right) and $B^{ \pm}$(left) candidates. The result of the lifetime fit is superimposed. The hatched areas represent the background content of the event samples.

\section{$6 \quad B^{0}$ mixing measurements}

Mixing allows the two neutral $B$ mesons in the $B^{0} \bar{B}^{0}$ coherent state to decay with the same flavor ( mixed events) or the opposite flavor (unmixed events). In a perfect detector one would then observe a time dependent oscillation in the rates of unmixed(+) and mixed(-) events :

$$
f_{ \pm}\left(\Delta t ; \Gamma, \Delta m_{d}\right)=\frac{1}{4} \Gamma \mathrm{e}^{-\Gamma|\Delta t|}\left[1 \pm \cos \Delta m_{d} \Delta t\right]
$$

where $\Delta m_{d}$ is the difference between the mass eigenstates $B_{H}^{0}$ and $B_{L}^{0}$. Due to imperfect tagging and vertex determination the observed rates become :

$$
\mathcal{F}_{ \pm}\left(\Delta t ; \Gamma, \Delta m_{d}, \mathcal{D}, \hat{a}\right)=\frac{1}{4} \Gamma \mathrm{e}^{-\Gamma|\Delta t|}\left[1 \pm \mathcal{D} \times \cos \Delta m_{d} \Delta t\right] \otimes \mathcal{R}(\Delta t ; \hat{a})
$$


where $\mathcal{D}$ is the dilution factor (section 4.2 ) and $\mathcal{R}$ is the $\Delta t$ resolution (section 4.3)

An unbinned maximum likelihood fit that takes into account individual event $\Delta t$ errors and tagging category is performed on events from the exclusively reconstructed $B^{0}$ sample (section 4.1), after tagging (section 4.2 ) has been performed. The value of $\Delta m_{d}$ is fitted simultaneously with the individual dilution factors for each tagging category. This information is later used in the $\sin 2 \beta$ extraction. Our preliminary result for $\Delta m_{d}$ is :

$$
\Delta m_{d}=0.516 \pm 0.031 \text { (stat.) } \pm 0.018 \text { (syst.) } \hbar \mathrm{ps}^{-1}
$$

A sample of events where a semileptonic $\left(D^{*} \ell \nu\right)$ instead of a hadronic $B^{0}$ decay has been reconstructed (7517 events) are analysed using the same method and fit. The preliminary result for $\Delta m_{d}$ from this sample is :

$$
\Delta m_{d}=0.508 \pm 0.020 \text { (stat.) } \pm 0.022 \text { (syst.) } \hbar \mathrm{ps}^{-1}
$$

Combining the $\Delta m_{d}$ results from the hadronic and semileptonic $B$ samples we obtain the preliminary result :

$$
\Delta m_{d}=0.512 \pm 0.017 \text { (stat.) } \pm 0.022 \text { (syst.) } \hbar \mathrm{ps}^{-1}
$$

The main sources of systematic errors are the $\Delta t$ resolution function, Monte Carlo statistics and the $B^{ \pm}$background in the semileptonic sample.

In an independent analysis a more abundant but less pure sample of dilepton events has been used. In this inclusive approach the mistag arising from cascade leptons and the $B^{ \pm}$fraction are extracted from the same fit as $\Delta m_{d}$. Our preliminary result for $\Delta m_{d}$ is :

$$
\Delta m_{d}=0.507 \pm 0.015 \text { (stat.) } \pm 0.022 \text { (syst.) } \hbar \mathrm{ps}^{-1}
$$

The results from the hadronic and dilepton samples are shown in Figure 4. The tagging performance parameters for each tagging method (category) are extracted from the fully reconstructed sample fits (hadronic and semileptonic) and are shown in Table 1.

\begin{tabular}{|l|c|c|c|}
\hline Tagging Category & $\varepsilon(\%)$ & $w(\%)$ & $Q(\%)$ \\
\hline \hline Lepton & $11.2 \pm 0.5$ & $9.6 \pm 1.7 \pm 1.3$ & $7.3 \pm 0.7$ \\
Kaon & $36.7 \pm 0.9$ & $19.7 \pm 1.3 \pm 1.1$ & $13.5 \pm 1.2$ \\
NT1 & $11.7 \pm 0.5$ & $16.7 \pm 2.2 \pm 2.0$ & $5.2 \pm 0.7$ \\
NT2 & $16.6 \pm 0.6$ & $33.1 \pm 2.1 \pm 2.1$ & $1.9 \pm 0.5$ \\
\hline \hline all & $76.7 \pm 0.5$ & & $27.9 \pm 1.6$ \\
\hline
\end{tabular}

Table 1: Tagging preformance parameters measured from the mixing maximum-likelihood fit for the fully-reconstructed $B^{0}$ sample. The uncertainties on $\varepsilon$ and $Q$ are statistical only.

\section{$7 \quad$ The $\sin 2 \beta$ measurement}

If one of the neutral $B$ mesons $\left(B_{t a g}\right.$ ) of the coherent $B^{0} \bar{B}^{0}$ pair decays to a definite flavor eigenstate at time $t_{t a g}$ and the other $B$ decays to a $C P$-even eigenstate like $J / \psi K_{S}^{0}$ or $\psi(2 S) K_{S}^{0}$ at time $t_{C P}$, 

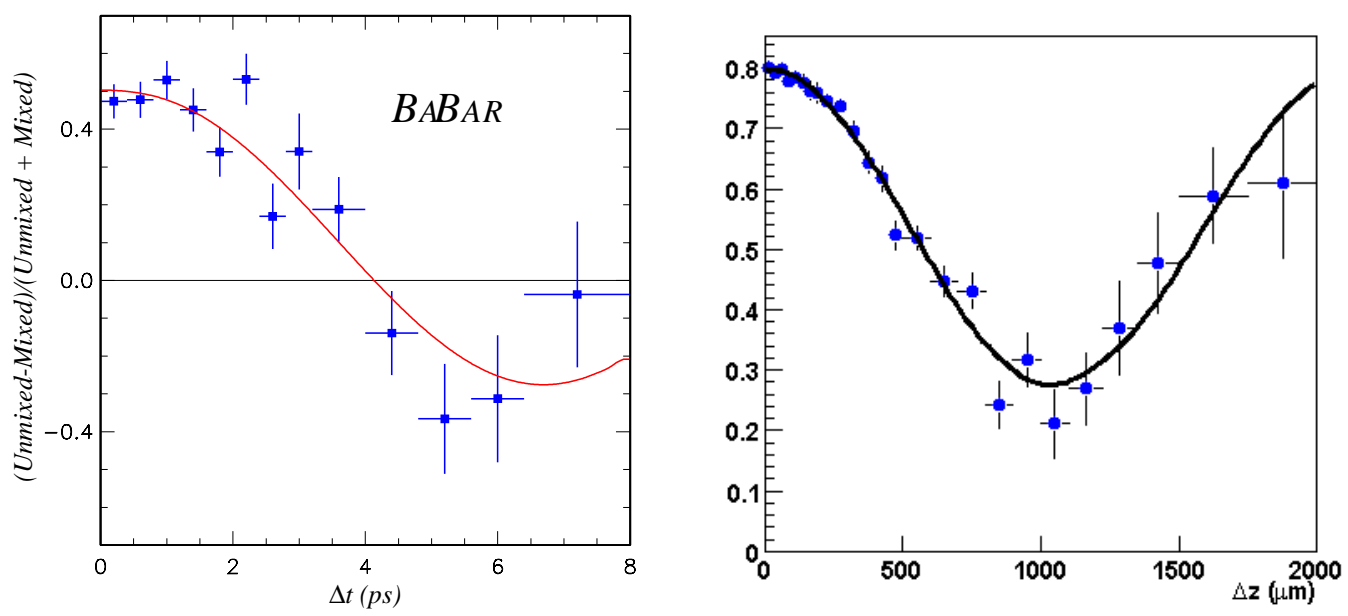

Figure 4: The observed time dependent asymmetries between unmixed and mixed events for the fully reconstructed (left) and dilepton (right) $B^{0}$ samples described in the text. The curves show the fit results.

then in a perfect detector the following decay rates would be observed :

$$
f_{ \pm}\left(\Delta t ; \Gamma, \Delta m_{d}, \sin 2 \beta\right)=\frac{1}{4} \Gamma \mathrm{e}^{-\Gamma|\Delta t|}\left[1 \pm \sin 2 \beta \times \sin \Delta m_{d} \Delta t\right]
$$

where $\Delta t=t_{C P}-t_{t a g}$ and the $(+)$ or $(-)$ sign indicates whether the $B_{t a g}$ is tagged as a $B^{0}$ or a $\bar{B}^{0}$ respectively. In the presense of the dilution factor $\mathcal{D}$ and $\Delta t$ resolution $\mathcal{R}$ the observed rates become :

$$
\mathcal{F}_{ \pm}\left(\Delta t ; \Gamma, \Delta m_{d}, \mathcal{D} \sin 2 \beta, \hat{a}\right)=\frac{1}{4} \Gamma \mathrm{e}^{-\Gamma|\Delta t|}\left[1 \pm \mathcal{D} \sin 2 \beta \times \sin \Delta m_{d} \Delta t\right] \otimes \mathcal{R}(\Delta t ; \hat{a})
$$

The time dependent decay rate asymmetry $\mathcal{A}_{C P}(\Delta t)$ is a $C P$-violating observable which (neglecting resolution effects) is approximately proportional to $\sin 2 \beta$ :

$$
\mathcal{A}_{C P}(\Delta t)=\frac{\mathcal{F}_{+}(\Delta t)-\mathcal{F}_{-}(\Delta t)}{\mathcal{F}_{+}(\Delta t)+\mathcal{F}_{-}(\Delta t)} \sim \mathcal{D} \sin 2 \beta \times \sin \Delta m_{d} \Delta t
$$

\subsection{Analysis procedure}

The extraction of $\sin 2 \beta$ from the data follows the following steps :

- Selection of the signal $B^{0} / \bar{B}^{0} \rightarrow J / \psi K_{S}^{0}$ and $B^{0} / \bar{B}^{0} \rightarrow \psi(2 S) K_{S}^{0}$ events, detailed in the following section. Backgrounds and in particular any admixture with the "wrong" $C P$ content have to be kept at a minimum level.

- Measurement of $\Delta t$. The resolution is studied using simulated events and its parameters are actually extracted from real data, as described in section 4.3.

- Determination of the flavor of the $B_{t a g}$, as described in section 4.2. The dilution factors $\mathcal{D}_{i}$ for each tagging category are measured on real data, as described in section 6 . 
- Extraction of the amplitude of the $C P$ asymmetry and the value of $\sin 2 \beta$ with an unbinned maximum likelihood fit, desribed in the following.

A blind analysis has been adopted for the extraction of $\sin 2 \beta$. A technique that hides the result of the fit by arbitrarily flipping its sign and adding an arbitrary offset, without affecting the error on the fitted parameters or their correlations, was used. Moreover, the visual $C P$ asymmetry in the $\Delta t$ distribution is hidden by multiplying $\Delta t$ by the sign of the tag and adding an arbitrary offset. Such an approach allows to optimise and finalise the event selection and fitting strategy as well as perform a variety of validation and stability checks without the posibility of any experimenter's bias.

\subsection{Event samples}

The $C P$ sample contains $B^{0}$ candidates reconstructed in the $C P$ eigenstates $J / \psi K_{S}^{0}$ or $\psi(2 S) K_{S}^{0}$. The charmonium mesons are reconstructed through their decays to $e^{+} e^{-}$and $\mu^{+} \mu^{-}$, while the $\psi(2 S)$ is also reconstructed through its decay to $J / \psi \pi^{+} \pi^{-}$. The $K_{S}^{0}$ is reconstructed through its decays to $\pi^{+} \pi^{-}$and $\pi^{0} \pi^{0}$.

Utilisation of the exclusively reconstructed $B$ samples (section 4.1) for the characterisation of the tagging and vertexing performance and quality has already been described. In addition 570 $B^{+} \rightarrow J / \psi K^{+}$candidates and $237 B^{0} \rightarrow J / \psi\left(K^{* 0} \rightarrow K^{+} \pi^{-}\right)$candidates have been reconstructed and used extensively in validation analyses.

\subsection{Selection of events in the $C P$ sample}

Events are required to have at least four reconstructed charged tracks, a vertex within $0.5 \mathrm{~cm}$ of the average position of the interaction point in the transverse plane, total visible energy greater than $5 \mathrm{GeV}$, and second-order normalized Fox-Wolfram moment[4] $\left(R_{2}=H_{2} / H_{0}\right)$ less than 0.5.

For the $J / \psi$ or $\psi(2 S) \rightarrow e^{+} e^{-}\left(\mu^{+} \mu^{-}\right)$candidates, at least one of the decay products is required to be positively identified as an electron (muon) in the EMC (IFR). Electrons outside the acceptance of the EMC are accepted if their $\mathrm{DCH} \mathrm{d} E / \mathrm{d} x$ information is consistent with the electron hypothesis. Looser particle identification criteria are applied on the second electron (muon) candidates. In the muon case, a minimum ionising signature in the EMC is required.

$J / \psi$ candidates are selected with an invariant mass greater than $2.95(3.06) \mathrm{GeV} / c^{2}$ for $e^{+} e^{-}$ $\left(\mu^{+} \mu^{-}\right)$and smaller than $3.14 \mathrm{GeV} / c^{2}$ in both cases. The $\psi(2 S)$ candidates in leptonic modes must have a mass within $50 \mathrm{MeV} / c^{2}$ of the $\psi(2 S)$ mass. The lower bound is relaxed to $250 \mathrm{MeV} / c^{2}$ for the $e^{+} e^{-}$mode. For the $\psi(2 S) \rightarrow J / \psi \pi^{+} \pi^{-}$mode, mass-constrained $J / \psi$ candidates are combined with pairs of oppositely charged tracks considered as pions, and $\psi(2 S)$ candidates with mass between $3.0 \mathrm{GeV} / c^{2}$ and $4.1 \mathrm{GeV} / c^{2}$ are retained. The mass difference between the $\psi(2 S)$ candidate and the $J / \psi$ candidate is required to be within $15 \mathrm{MeV} / \mathrm{c}^{2}$ of the known mass difference.

$K_{S}^{0}$ candidates reconstructed in the $\pi^{+} \pi^{-}$mode are required to have an invariant mass, computed at the vertex of the two tracks, between $486 \mathrm{MeV} / c^{2}$ and $510 \mathrm{MeV} / c^{2}$ for the $J / \psi K_{S}^{0}$ selection, and between $491 \mathrm{MeV} / c^{2}$ and $505 \mathrm{MeV} / c^{2}$ for the $\psi(2 S) K_{S}^{0}$ selection.

For the $J / \psi K_{S}^{0}$ mode we also consider the decay of the $K_{S}^{0}$ into $\pi^{0} \pi^{0}$. For pairs of $\pi^{0}$ candidates with total energy above $800 \mathrm{MeV}$ we determine the most probable $K_{S}^{0}$ decay point along the path defined by the $K_{S}^{0}$ momentum vector and the primary vertex of the event. The decay-point probability is the product of the $\chi^{2}$ probabilities for each photon pair constrained to the $\pi^{0}$ mass. We require the distance from the decay point to the primary vertex to be between $-10 \mathrm{~cm}$ and $+40 \mathrm{~cm}$ and the $K_{S}^{0}$ mass measured at this point to be between 470 and $536 \mathrm{MeV} / \mathrm{c}^{2}$. 
$B_{C P}$ candidates are formed by combining mass-constrained $J / \psi$ or $\psi(2 S)$ candidates with massconstrained $K_{S}^{0}$ candidates. Cuts on the colinearity of flight vertex and momentum of the $K_{S}^{0}$ (for $\pi^{+} \pi^{-}$decays), the cosine of the helicity angle of the $J / \psi$ or $\psi(2 S)$ in the $B$ candidate rest frame $\left(e^{+} e^{-}\right.$and $\mu^{+} \mu^{-}$modes) or the cosine of the angle between the $B_{C P}$ candidate three-momentum vector and the thrust vector of the rest of the event $\left(\psi(2 S) \rightarrow J / \psi \pi^{+} \pi^{-}\right.$mode) are applied to achieve the required signal purity.

$B_{C P}$ candidates are identified in the $m_{\mathrm{ES}}-\Delta E$ plane (see section 4.1). Signal event yields and purities, determined from a fit to the $m_{\mathrm{ES}}$ distributions after selection on $\Delta E$, are presented in Table 2. The $C P$ candidate events are 168 with a purity of $95.6 \%$. In 120 of these events there is information on the flavor of the other $B$. These events are used in the final fit for $\sin 2 \beta$. Distributions of $\Delta E$ and $m_{\mathrm{ES}}$ are shown in Figures 5 and 6.

\begin{tabular}{|l|l|c|c|}
\hline Final state & All events & Purity & Tagged events \\
\hline \hline$J / \psi K_{S}^{0}\left(K_{S}^{0} \rightarrow \pi^{+} \pi^{-}\right)$ & 124 & $96 \%$ & 85 \\
$J / \psi K_{S}^{0}\left(K_{S}^{0} \rightarrow \pi^{0} \pi^{0}\right)$ & 18 & $91 \%$ & 12 \\
$\psi(2 S) K_{S}^{0}$ & 27 & $93 \%$ & 23 \\
\hline \hline
\end{tabular}

Table 2: Event yields for the $C P$ samples used in this analysis. The total number of events in the $m_{\mathrm{ES}}-\Delta E$ signal box and their purity, as well as the size of the subsamples where the other $B$ is tagged, are shown.

\subsection{Extracting $\sin 2 \beta$}

The $\Delta t$ of the 120 selected and tagged events is fitted to the expected time evolution (equation 5 ) with an unbinned maximum likelihood fit. Individual event errors on $\Delta t$ are taken into account. The resolution determined on the fully reconstructed sample and the mistag factor $w_{i}$ corresponding to the tagging category for each event are used in the fit. The $\Delta m_{d}$ are fixed to the nominal PDG [5] values of $\tau_{B^{0}}=1.548 \mathrm{ps}$ and $\Delta m_{d}=0.472 \hbar \mathrm{ps}^{-1}$ respectively. The resulting errors on $\sin 2 \beta$ due to these uncertainties are 0.002 and 0.015 .

\subsection{Fit validation, systematics studies and null $C P$ tests}

Knowledge of the mistag fractions, description of the $\Delta t$ resolution and backgrounds are (in that order) the main sources of systematic errors. All these have been extracted from real data. Real data, fully simulated Monte Carlo, and "Toy" Monte Carlo samples have been used to validate the method and implementation of the fit, to rule out possible biases from the method itself, and to assess the size of systematic errors.

The full CP analysis and fit were performed on data samples that have no $C P$ asymmetry. No significant apparent $C P$ effect was measured, as shown in Table 3 . The $1.9 \sigma$ asymmetry in the $J / \psi K^{* 0}$ channel is interpreted as a statistical fluctuation. 

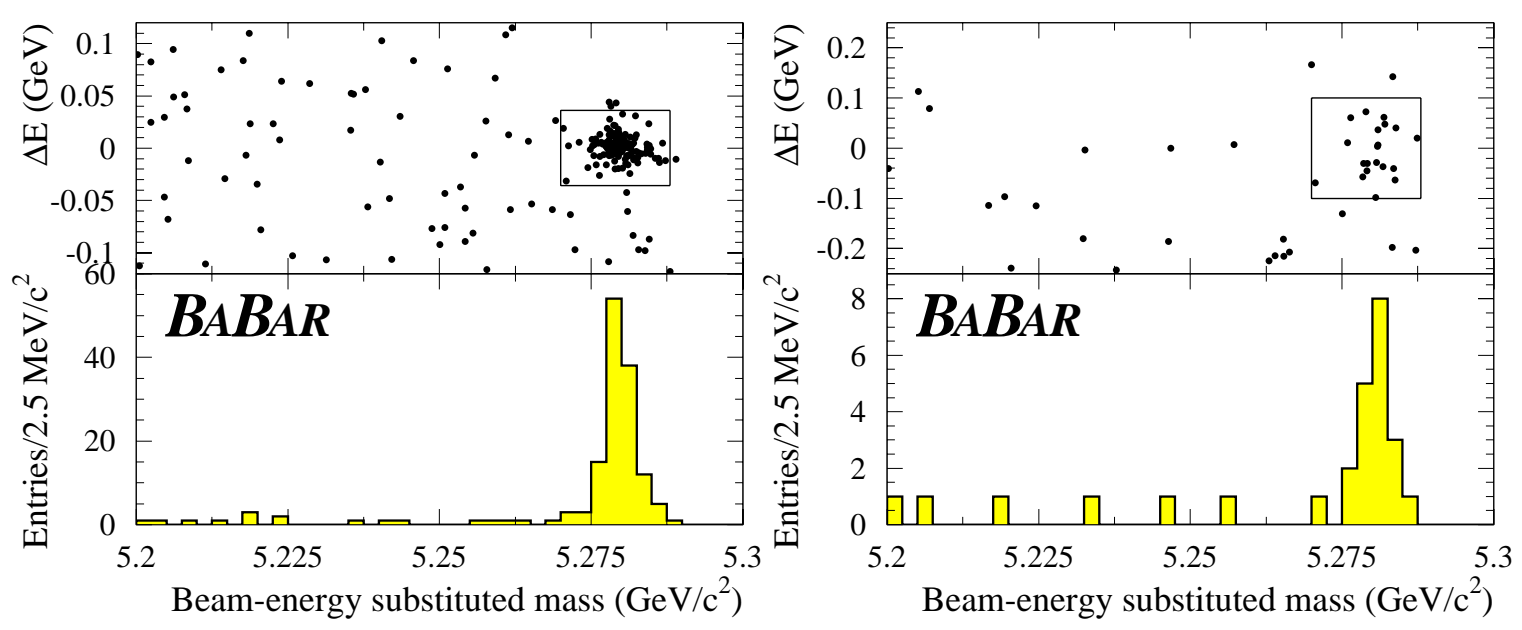

Figure 5: $J / \psi K_{S}^{0}$ signal. Left: $K_{S}^{0} \rightarrow \pi^{+} \pi^{-}$, Right: $K_{S}^{0} \rightarrow \pi^{0} \pi^{0}$

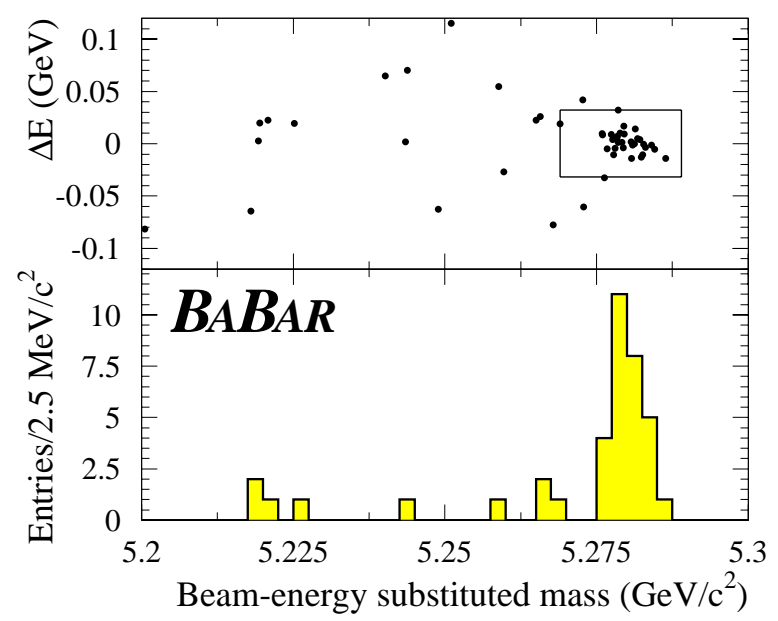

Figure 6: $\psi(2 S) K_{S}^{0}\left(K_{S}^{0} \rightarrow \pi^{+} \pi^{-}\right)$signal.

\begin{tabular}{|l|c|}
\hline Sample & Apparent $C P$-asymmetry \\
\hline \hline Hadronic charged $B$ decays & $0.03 \pm 0.07$ \\
\hline Hadronic neutral $B$ decays & $-0.01 \pm 0.08$ \\
\hline$J / \psi K^{+}$ & $0.13 \pm 0.14$ \\
\hline$J / \psi K^{* 0}\left(K^{* 0} \rightarrow K^{+} \pi^{-}\right)$ & $0.49 \pm 0.26$ \\
\hline
\end{tabular}

Table 3: Results of fitting for apparent $C P$ asymmetries in various charged or neutral flavoreigenstate $B$ samples. 


\subsection{Results}

The maximum-likelihood fit for $\sin 2 \beta$ on the full tagged sample of $B^{0} / \bar{B}^{0} \rightarrow J / \psi K_{S}^{0}$ and $B^{0} / \bar{B}^{0} \rightarrow$ $\psi(2 S) K_{S}^{0}$ events yields the preliminary result :

$$
\sin 2 \beta=0.12 \pm 0.37 \text { (stat) } \pm 0.09 \text { (syst) }
$$

The results of the fit for each type of $C P$ sample and for each tagging category are given in Table 4 . The contributions to the systematic uncertainty are summarized in Table 5 . The $\Delta t$ distributions for $B^{0}$ and $\bar{B}^{0}$ tags are shown in Fig. 7 and the raw asymmetry as a function of $\Delta t$ is shown in Fig. 8. The probability of obtaining a value of the statistical error larger than the one we observe is estimated at $5 \%$. Based on a large number of full Monte Carlo simulated experiments with the same number of events as our data sample, we estimate that the probability of finding a lower value of the likelihood than our observed value is $20 \%$.

\begin{tabular}{|l|c|}
\hline sample & $\sin 2 \beta$ \\
\hline \hline$C P$ sample & $\mathbf{0 . 1 2} \pm \mathbf{0 . 3 7}$ \\
\hline$J / \psi K_{S}^{0}\left(K_{S}^{0} \rightarrow \pi^{+} \pi^{-}\right)$events & $-0.10 \pm 0.42$ \\
other $C P$ events & $0.87 \pm 0.81$ \\
\hline Lepton & $1.6 \pm 1.0$ \\
Kaon & $0.14 \pm 0.47$ \\
NT1 & $-0.59 \pm 0.87$ \\
NT2 & $-0.96 \pm 1.30$ \\
\hline
\end{tabular}

Table 4: $\sin 2 \beta$ fit results from the entire $C P$ sample and various subsamples.

\begin{tabular}{|l|c|}
\hline Source of uncertainty & Uncertainty on $\sin 2 \beta$ \\
\hline \hline uncertainty on $\tau_{B}^{0}$ & 0.002 \\
uncertainty on $\Delta m_{d}$ & 0.015 \\
uncertainty on $\Delta z$ resolution for $C P$ sample & 0.019 \\
uncertainty on time-resolution bias for $C P$ sample & 0.047 \\
uncertainty on measurement of mistag fractions & 0.053 \\
different mistag fractions for $C P$ and non- $C P$ samples & 0.050 \\
different mistag fractions for $B^{0}$ and $\bar{B}^{0}$ & 0.005 \\
background in $C P$ sample & 0.015 \\
\hline \hline total systematic error & $\mathbf{0 . 0 9 1}$ \\
\hline
\end{tabular}

Table 5: Summary of systematic uncertainties on $\sin 2 \beta$ 


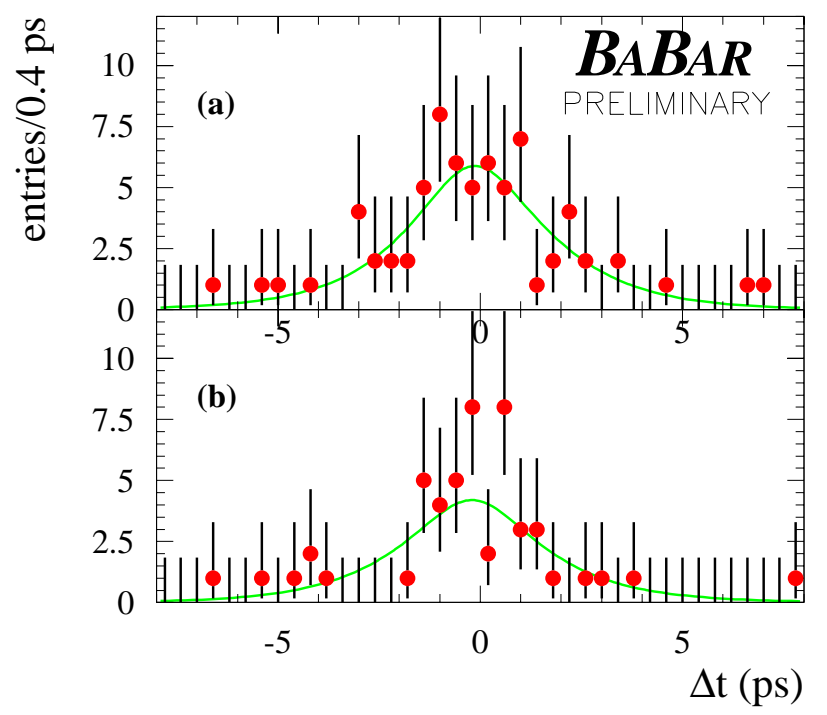

Figure 7: Distribution of $\Delta t$ for (a) the $B^{0}$ tagged events and (b) the $\bar{B}^{0}$ tagged events in the $C P$ sample. The error bars plotted for each data point assume Poisson statistics. The curves correspond to the result of the unbinned maximum-likelihood fit and are each normalized to the observed number of tagged $B^{0}$ or $\bar{B}^{0}$ events.

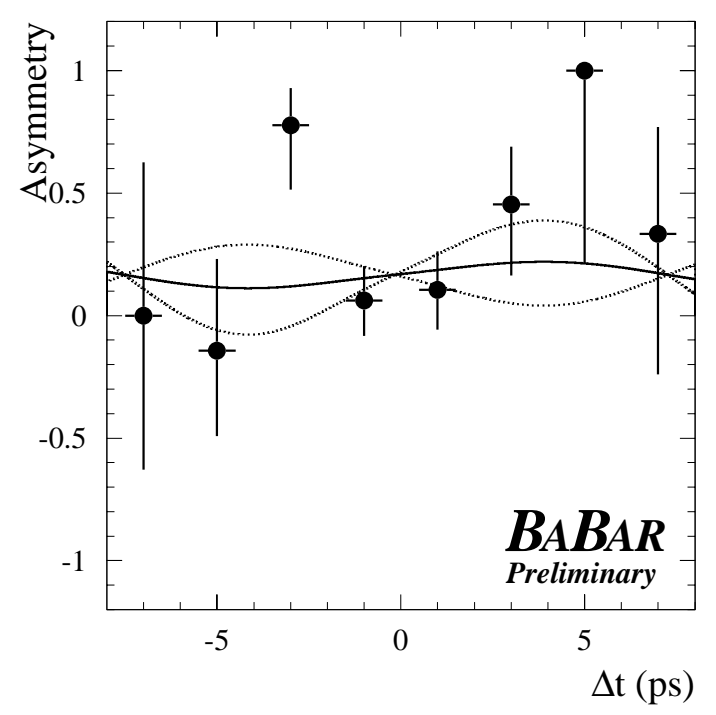

Figure 8: The raw $B^{0}-\bar{B}^{0}$ asymmetry $\left(N_{B^{0}}-N_{\bar{B}^{0}}\right) /\left(N_{B^{0}}+N_{\bar{B}^{0}}\right)$ with binomial errors as function of $\Delta t$. The solid curve represents our central value of $\sin 2 \beta$. The two dotted curves correspond to one statistical standard deviation from the central value. The curves are not centered at $(0,0)$ in part because the probability density functions are normalized separately for $B^{0}$ and $\bar{B}^{0}$ events, and our $C P$ sample contains an unequal number of $B^{0}$ and $\bar{B}^{0}$ tagged events $\left(70 B^{0}\right.$ versus $\left.50 \bar{B}^{0}\right)$. The $\chi^{2}$ between the binned asymmetry and the result of the maximum-likelihood fit is 9.2 for 7 degrees of freedom. 


\section{Conclusions and prospects}

The first $B A B A R$ measurement of the $C P$-violating asymmetry parameter $\sin 2 \beta$ has been presented :

$$
\sin 2 \beta=0.12 \pm 0.37 \text { (stat) } \pm 0.09 \text { (syst) (preliminary) }
$$

BABAR aims at collecting more than $20 \mathrm{fb}^{-1}$ of data by the end of Run 1 in fall 2000. A measurement of $\sin 2 \beta$ with a precision better than 0.2 is expected early in 2001 .

Very competitive preliminary results have also been presented for the $B$ meson lifetimes, as well as the first measurements of $B^{0} \bar{B}^{0}$ mixing at the $\Upsilon(4 S)$. These measurements will also benefit in the near future from the expected significant increase in statistics.

\section{References}

[1] C. Jarlskog, in CP Violation, C. Jarlskog ed., World Scientific, Singapore (1988).

[2] BABAR Collaboration, B. Aubert et al., "The first year of the BABAR experiment at PEPII", BABAR-CONF-00/17, submitted to the XXX ${ }^{\text {th }}$ International Conference on High Energy Physics, Osaka, Japan.

[3] ARGUS collaboration, H. Albrecht et al., Z Phys. C48, 543 (1990).

[4] G. C. Fox and S. Wolfram, Phys. Rev. Lett. 41, 1581 (1978).

[5] Particle Data Group, D. E. Groom et al., Eur. Phys. Jour. C 15, 1 (2000). 